\title{
EL FORCEPS DE KIELLAND EN EL MANEJO DE LAS PRESENTACIONES OCCIPITOPOSTERIORES PERSISTENTES
}

\author{
Dr. Jaime Díaz Garzón*
}

Indudablemente una complicación relativamente frecuente $(6 \%),(1)$ $y$ que en muchas ocasiones acarrea gran peligro tanto para el niño como para la madre, es la "detención" que ocurre por "falta de rotación" en las presentaciones

occípitoposteriores (ODP-OIP). Los mecanismos por los cuales esto sucede no son bien conocidos, pero parece que hay varios factores que intervienen en su producción. Entre otros podrían citarse la estrechez pélvica en el diámetro transverso (como en la pelvis androide $y$ antropoide), convergencia anormal de las paredes pélvicas, un sacro poco cóncavo, espinas ciáticas prominentes. También se aduce que la hipodinamia uterina, dificultades en el borramiento y dilatación del cuello, la ruptura prematura de membranas, una placenta baja implantada anteriormente, una inclinación pélvica defectuosa, una presentación deflejada, una dextrorrotación exagerada, etc. podrían conducir a una falta de rotación en las presentaciones occípitoposteriores.

El ideal sería "permitir y favorecer" que el polo cefálico hiciera su recorrido gracias a una buena dinámica uterina, un buen moldamiento cefálico — cuando es necesario-, sin tener que recurrir a métodos direc- tos más 0 menos traumatizantes. Pero hemos visto que esta actitud pasiva puede ser arriesgada por el gran peligro que pueden correr la madre $y$ el niño.

Muchos métodos y sistemas han sido descritos para la corrección de este problema. Smellie, hacia 1750 describió una maniobra rotadora, usando sus pequeños fórceps rectos. También cabe destacar los métodos de Holland, de Pomeroy; (2) las maniobras de Scanzoni (3), Ramírez M. (4), la de "Ilave en la cerradura" de De Lee; el empleo de fórceps especiales como el de Barton, Kielland, etc. Todos estos métodos son más o menos buenos y el resultado dependerá de la adecuada escogencia de los casos ( $\sin$ duda hay algunos que deben ser terminados por cesárea) y de la habilidad particular del obstetra. Con uno o dos de estos métodos que el especialista conozca perfectamente, es suficiente para la adecuada atención del parto.

Nuestro interés fue el de comprobar la bondad del fórceps de KieIland para este tipo de problema. Es bien sabido que para muchos autores (5), el empleo de estos fórceps

* Ex-Instructor del Departamento de Obstetricia y Ginecología de la Universidad Nacional. 
es el método de elección para la corrección de las presentaciones occípitoposteriores persistentes (OPP).

El procedimiento fue realizado en 30 casos en el Instituto Materno Infantil de Bogotá, por miembros del personal docente del Departamento de Obstetricia y Ginecología de la Universidad Nacional, y por personal en adiestramiento. Fueron escogidas aquellas pacientes que llevaban 1 hora 0 más en el segundo período del parto, y de que a pesar de tener una buena dinámica uterina $y$ de haberse intentado en ellas la rotación digital, no había sido posible la corrección del problema.

De las 30 pacientes, 25 (83,3\%) fueron primigestantes, y el resto multíparas. Todas con embarazo a término y feto vivo. Como método anestésico se usó Pentothal en $12(40 \%)$, anestesia peridural en $14(46,6 \%)$ y bloqueo pudendo en $4(13,3 \%)$.

Veintitrés fueron ODP y el resto (7) OIP.

En 5 la aplicación fue alta "fórceps Alto" ( 2 en primigestantes y 3 en multíparas). En 14 fué en el estrecho medio "fórceps medio" y 11 en el estrecho inferior "fórceps bajo".

El procedimiento de aplicación del fórceps fue sencillo. Para explicarlo tomemos como ejemplo una ODP:

Se hizo un proyecto de toma para la variedad anterior opuesta (OIA), procediéndose a hacer la aplicación directa de la cuchara izquierda, tomando el mango con la mano izquierda y guiando la cuchara con la mano derecha. La cuchara derecha o anterior se aplicó en la región parieto-malar izquierda, siendo guiada por la mano izquierda. La aplicación de las cucharas fue "directa", ini- ciándose la aplicación estando el mango de la rama en contacto con el muslo opuesto, llevándose hacia la parte media a medida que la cuchara iba entrando en la cavidad pélvica. La articulación de las cucharas se realizó sin complicaciones y se procedió a practicar la rotación "como llave en la cerradura", sin hacer traslación de los mangos en el espacio y centrando los picos de las cucharas. No se intentó la tracción al mismo tiempo de la rotación ni ésta se hizo durante la contracción uterina. La orientación del fórceps y su rotación fue diferente según la toma fuera "alta" o fuera en estrecho medio o bajo.

En caso de toma alta, el fórceps se puso invertido y la rotación se hizo en 2 tiempos: primero se rotó de ODP a ODA (90\%) y luego se encajó y descendió la presentación (traccionando abajo y atrás) por el diámetro oblicuo derecho; estando ya el vértice en piso perineal, se terminó la rotación llevando el occipital contra el pubis y después halando abajo y adelante, se reflejó la cabeza para lograr su extracción. Es interesante notar que estando la presentación alta, y habiendo necesidad de descenderla traccionando en el eje de la pelvis (imaginariamente una línea que vaya del ombligo al coxis), es necesario colocar el fórceps invertido, para que al rotar a ODA quede al derecho y se pueda aprovechar su curva en bayoneta, lo cual facilita la tracción abajo y atrás, deprimiendo con los mangos el periné (recordar episiotomía en caso necesario).

Por otro lado, cuando la presentación estaba detenida a nivel del estrecho medio o del inferior, la rotación se hizo de una vez a OP (rechazándola o descendiéndola previamente un poco, según el caso), y en cuanto a que los fórceps estuvieran 
invertidos o al derecho, no tuvo importancia.

Para las OIP, lógicamente se hizo la toma como para ODA, haciéndose la aplicación de las cucharas de un modo similar al ya descrito, teniéndose en cuenta lo antes dicho para las ODP.

En 4 casos en los cuales la aplicación de la cuchara anterior fue un poco difícil- aún empleando el método de la aplicación directa-, se rotó digitalmente la presentación a OS, haciéndose la toma muy sencilla como para OP, procediéndose a la rotación etc.

\section{Resultados}

Tanto desde el punto de vista materno como fetal, los resultados fueron buenos.

En un niño se presentó parálisis facial-pasajera (desapareció en 48 horas). El resto de los niños no tuvo ninguna complicación.

En 5 pacientes (todas primigestantes) $(16,6 \%)$, se produjeron desgarros cérvico-vaginales (uno de ellos más o menos extenso) que se suturaron sin ningún problema. El resto de las pacientes no tuvo complicaciones.

En un caso (primigestante, feto de 3.200 grs.), la rotación no se pudo realizar, haciéndose la extracción en OS.

\section{Comentarios}

Debido a sus características de ser liviano y angosto, de casi no tener curvatura pélvica y de tener una curva "bayoneta", se puede decir que el fórceps de Kielland es ideal cuandoquiera que se necesite realizar rotaciones, y también -aunque esto úl- timo en la actualidad sólo se hace excepcionalmente- en las aplicaciones altas (6). Gracias a ser un instrumento prácticamente recto es posible hacer la maniobra de Scanzoni - modificada_, sin necesidad de retirarlo y hacer una segunda toma, como ya lo había preconizado Smellie usando sus fórceps rectos. Algunos autores aducen que esta maniobra podría causar grandes desgarros cérvicovaginales. Nosotros tuvimos un $16,6 \%$ de desgarros, que si se compara con otras series $(14,8 \%)$ (7) en que se usaron otros métodos de rotación, vemos que los resultados son similares.

Para la aplicación de la cuchara anterior nosotros no empleamos el método prescrito por Kielland (8), por parecernos muy peligroso, sino más bien la maniobra de $M$. La Chapelle, o mejor aún "la aplicación directa".

Es necesario hacer notar que, en términos generales, toda maniobra de rotación "debe hacerse o bien por encima (rechazando suavemente la presentación hacia arriba) o por debajo (descendiéndola) del plano en que ocurrió la detención". Además hay que llamar la atención, y también de un modo general, sobre el hecho de que estando ya las cucharas aplicadas, cuando hay dificultades en la rotación y sin embargo se insiste, posiblemente la madre está siendo lesionada, y cuando hay dificultad a la tracción es el feto el que está siendo lesionado.

\section{Resumen y Conclusiones}

1) Se empleó el fórceps de Kielland en 30 casos de presentación occípito-posterior persistente (OPP).

2) Los resultados materno fetales pueden considerarse como buenos. 
3) Es evidente que el fórceps de Kielland por no tener curvatura pélvica, facilita grandemente las maniobras de rotación, y por su "curva en bayoneta", la tracción en tomas altas.

4) Así mismo facilita la maniobra de Scanzoni - modificada-, pues no se necesita hacer una segunda toma (maniobra de Smellie).

5) No se consideró sus ventajas en casos de detenciones transversas profundas (ODT-OIT), o de asinclitismo.

\section{Summary}

1) Kielland forceps were used in 30 cases with persistent occipito-posterior presentations (R.O.P. and L.O.P.).

2) The results for both mother and inflant were good.

3) The Kielland forceps without the pelvic curve facilitates greatly the procedure of rotation and with its "bayonet curve" gives traction to the higher areas.

4) The use of Kielland forceps facilitates the Scanzoni modified ma- neuver, and it is not necessary to use a second application. (Smellie's Maneuver).

5) The advantages in cases of detained "transverse lies" are not considered, nor asinclitism.

de sfscG,

\section{BIBLIOGRAFIA}

1 WILLIAMS, Obstetrics, 13th Ed. 1966 pp. 839.

2 ANDERSON, DAVID G. "Arrested occipit posterior positions". Clinical Obst. \& Gynec. 8 : $867,1965$.

3 DANFORTH, D. N., Method of forceps rotation in persistent occiput posterior. Amer. J. Obst. \& Gynec. 65: 120, 1953.

4 RAMIREZ MERCHAN R., Rev. Soc. Col. ObsGin., Vol. 1 № 2.

5 JONES, E. P., Kiellands forceps, Butterworth, London, 1952.

6 MEJIA VIEIRA, A., EI fórceps de Kielland. Tesis de grado, 1942.

7 KING, E. L., HERRING, J. S., Modification of the Scanzoni rotation in the management of persistent occipitoposterior positions. Amer. J. Obst. \& Gynec. 61: 872, 1951.

8 WILSON, J. R., Atlas of Obstetric technic. Mosby, St. Louis. 1961, pp. 136. 